\title{
How do taxes, benefits and public spending evolve for a taxpayer during their lifetime?
}

\author{
François Lévesque* \\ Polytechnique Montréal
}

\author{
Luc Godbout ${ }^{\dagger}$ \\ Michaël Robert-Angers ${ }^{\dagger}$ \\ Chaire de recherche en fiscalité et en finances publiques \\ Université de Sherbrooke
}

\author{
Thomas Hurtut \\ Polytechnique Montréal
}

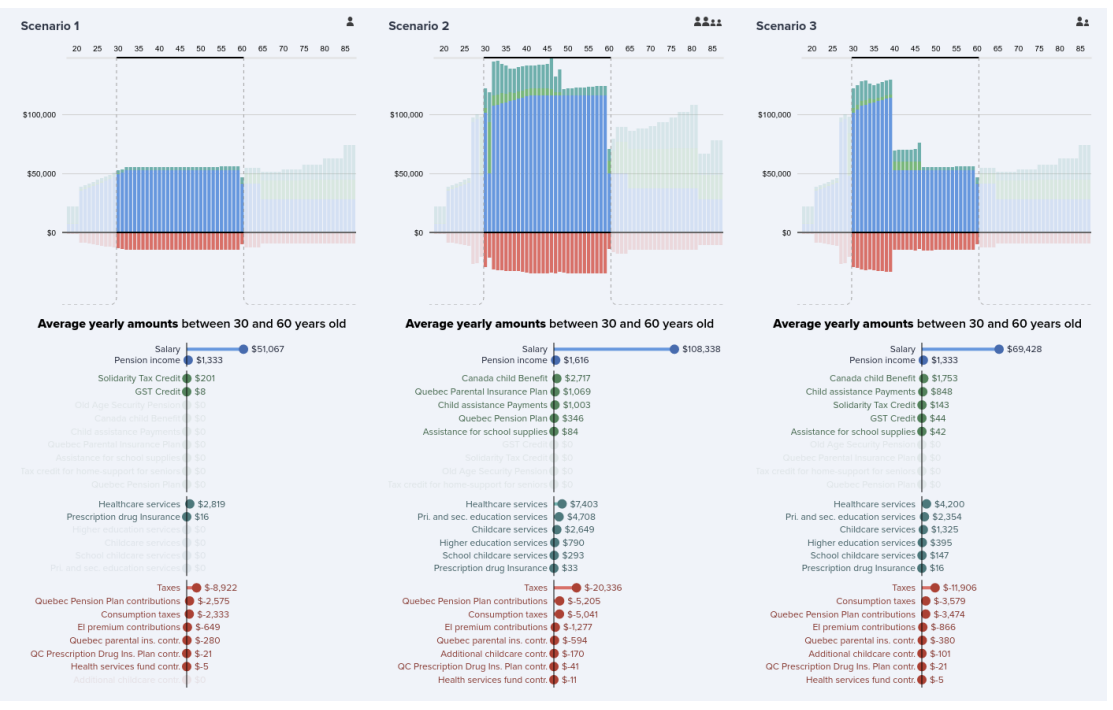

Figure 1: A narrative step in the presented visual case study, comparing three life stories in terms of tax levies and public services.

\section{Visual Case Study}

We present a visual case study conducted by the Research Chair in Taxation and Public Finance from Sherbrooke University, visually designed and developed in collaboration with Polytechnique Montreal. Initally published in French, an English version is available online at https://life-stories.netlify.app/. One of five goals of the aforementionned research chair is to disseminate its research results to a general public audience, and more specifically Quebec citizens. The project presented in this case study participates to that objective.

General Description How do taxes, benefits and public spending evolve for a taxpayer during their lifetime? This project explores a narrative format to make a possible answer accessible to a general audience. It is based on a thorough analysis of both the tax levies (including consumption taxes), as well as tax benefits and the monetary value of public services (national education, healthcare, childcare etc.) obtained over a lifespan for a Quebec province household [3]. This analysis showed that three different average life stories, depending on the life events that can affect an household (studies, changes in the size and composition of the household etc.), are reasonable ways to give representative and accessible answers to the title question.

Design Considerations Based on these three life stories, we investigated a data-driven visual story based on three parallel visualizations combined to a scrollytelling interaction [2], which has

*francois.levesque@polymtl.ca

†1uc.godbout@usherbrooke.ca, michael.robert-angers@usherbrooke.ca

†thomas.hurtut@polymtl.ca proven to be a powerful narrative format to organize and convey complex information [1]. This design is responsive and therefore slightly differs on a vertical ratio screen (e.g. on cellphones). On a horizontal desktop screen (Figure 1), each life story is vertical and composed of two linked visualizations. In the upper panel is a histogram of the household income and public services (positive side), and tax levies (negative side), over time. In the down panel, a horizontal bar chart shows the precise decomposition of each category from the above histogram. Scrolling controls the timeline, life being decomposed into key periods of time (studying, parenting, etc.). Scrolling also makes small paragraph of text elements hovering the view, bringing some explanations and insights about the situation. Near the end of the narrative format, cumulative histograms and an self-exploration view with interactive controls are also offered to the reader. On small vertical screens, the bar chart based decomposition of categories, and the exploration tool are omitted, and the layout of the three histograms is vertical.

Public reception This visualization format has been visited by around 2000 unique visitors during the month following its release. It has also been covered in at least three daily newspapers and magazines.

\section{REFERENCES}

[1] R. Kosara and J. Mackinlay. Storytelling: The next step for visualization. Computer, 46(5):44-50, 2013.

[2] B. Lee, N. H. Riche, P. Isenberg, and S. Carpendale. More than telling a story: Transforming data into visually shared stories. IEEE computer graphics and applications, 35(5):84-90, 2015.

[3] M. Robert-Angers, A. Kerkhoff, A. Genest-Grégoire, and L. Godbout. Récits de vie - document accompagnateur. Technical report, Cahier de recherche 2020-05, CFFP, Mar. 2020. 